Originalartikel

\title{
Zur Anwendung eines elektronischen Bienenzählgerätes am Flugloch eines Bienenvolkes
}

\author{
M. Rickli ${ }^{1}$, G. Bühlmann ${ }^{1}$, L. Gerig ${ }^{1}$, H. Herren ${ }^{2}$, H.J. Schürch ${ }^{2}$, \\ W. Zeier ${ }^{3}$ und A. Imdorf ${ }^{1 *}$
}

1 Eidgenössische Forschungsanstalt für Milchwirtschaft, Sektion Bienen, CH-3097 Liebefeld, Schweizenn;

${ }_{2}^{2}$ Abteilung Forschung und Entwicklung, GD PTT, Schweizenn;

3 Ingenieurschule Bern, Schweizenn.

(eingegangen 30.August 1988, angenommen 20.April 1989)

Zusammenfassung - Die Flugaktivität kann zusammen mit Erhebungen der Volkstärke und der Brutfläche bedeutende Kriterien zur Untersuchung von Entwicklung und Zustand eines Bienenvolkes liefern. Zur Dauerüberwachung der Aktivitäl wurde das Flugoch eines freifliegenden Volkes in 16 Kanäle unterteilt, von denen jeder mit zwei parallelen Lichtschranken ausgerüstet wurde. Damit wurden Ausgänge und Eingänge der Bienen erkannt und registriert.

Für 1986 und 1987 liegen die täglichen Aktivitätsprofile mit einer Auflösung von 10 min vor. In dieser Publikation werden die Möglichkeiten des Zählgerätes anhand von Beispielen beschrieben. Für die uns interessierende Ermittlung der täglichen Sterberate, die sich aus der Bilanz "Ausgänge minus Eingänge" ergibt, ist das Zählgerät zu ungenau, da hier jede einzelne Biene registriert werden muss. Um Aktivitätsverläufe oder -veränderungen zu untersuchen, eignet es sich jedoch sehr gut.

Apis mellifera - Actograph — Zählgerät — Flugaktivität — Volksstärke

\section{Einleitung}

Verschiedene Methoden zur quantitativen Ertassung der Aktivität eines Bienenvolkes kamen bisher zur Anwendung : Wägen (z.B. Koulichkov, 1971), Wägen der ein- und ausfliegenden Bienen (Chauvin, 1976), Abhören der Geräuschentwicklung (Dietlein, 1985), Rückschlüsse aus dem Rückbehalt von Pollenfallen (z.B. Free und Preece, 1969; Hirschfelder, 1951), stichprobenweises Zählen der einund/oder ausfliegenden Bienen (Gary, 1967), Verschliessen des Flugloches und Zählen der heimkehrenden Bienen (z.B. Robinson und Morse, 1982; Danka et al., 1986; Danka und Gary, 1987), photoelektrisches Erfassen mit oder ohne Trennung von Einflug und Ausflug (Burrill und Dietz,

\footnotetext{
* Korrespondierender Autor
} 
1973; Erickson et al., 1973; Spangler, 1969; Spangler und Takessian, 1984; Verbeek und Verbeek, 1974).

Wir haben ein Gerät mit Infrarot-Lichtschranken und elektronischer Datenerfassung zur Dauerüberwachung der Flugaktivität eines freifliegenden Bienenvolkes entwickelt. Um die Flugaktivität, rsp. die Ein- und Ausgänge am Flugloch unter naturnahen Bedingungen zu erfassen, wurde darauf geachtet, dass möglichst wenig Eingriffe wie Verengung des Flugloches oder Markierung von einzelnen Bienen notwendig waren. Zielsetzung neben der kompletten Erfassung der Flugaktivität war, die Sterberate von adulten Bienen zu erfassen. In Liebefeld werden seit 1969 routinemässig Erhebungen der Stärke aller Völker durchgeführt (Imdorf et al., 1987). Die Anzahl adulter Bienen, die Flächen an offener und an gedeckelter Brut werden im Abstand von 3 Wochen bestimmt (= Populationsmessungen). Daraus lässt sich die Sterberate errechnen (Bühlmann, 1985; Gerig und Bühlmann, 1985). Die Bilanz des Zähigerätes "Ausflug minus Einflug» am Ende eines Tages sollte darüber Auskunft geben, wie viele Bienen ausgeflogen, aber nicht mehr ins Volk zurückgekehrt sind. Weitere Möglichkeiten der Anwendung des Gerätes sind : Überprüfen des Einflusses von Klimaveränderungen, von Änderungen im Nahrungsangebot, von Pestiziden und Krankheiten auf Sterberate und Flugaktivität.

Eine Übersicht über die täglichen Aktivitätsprofile des Jahres 1986 wurde von Bühlmann et al. (1987) veröffentlicht. Ziel dieser Arbeit ist, Möglichkeiten und Grenzen des Gerätes anhand von "normalen" und besonderen Flugtagen zu bestimmen.

\section{Methoden}

\section{Erfassen der Eingänge und Ausgänge}

Das normale Flugloch eines freifliegenden Bienenvolkes wurde in 16 gleich grosse Kanäle unterteilt $(8 \times 8 \times 7 \mathrm{~mm}$; Abb. 1). Diese Unterteilung lässt Bienen, Drohnen und Königin genügend Platz, so dass sich bei hoher Aktivität kaum Behinderungen ergeben. Jeder Kanal wurde mit zwei im Abstand von $2,53 \mathrm{~mm}$ horizontal montierten Infrarot-Lichtschranken versehen, damit die Bewegungsrichtung der passierenden Biene erkannt wurde (Abb. 1b; Wellenlänge $940 \mathrm{~nm}$, licht-emittierende Dioden TIL 24 und Phototransistoren LS 618, beide von Texas Instruments). Infrarot-Lichtschranken wurden gewählt, weil die Bienen dadurch in ihrem Verhalten nicht gestört werden. Mit dem Einbau der Lichtschranken in die Kanäle wurde eine Störung durch Fremdlicht unwahrscheinlich.

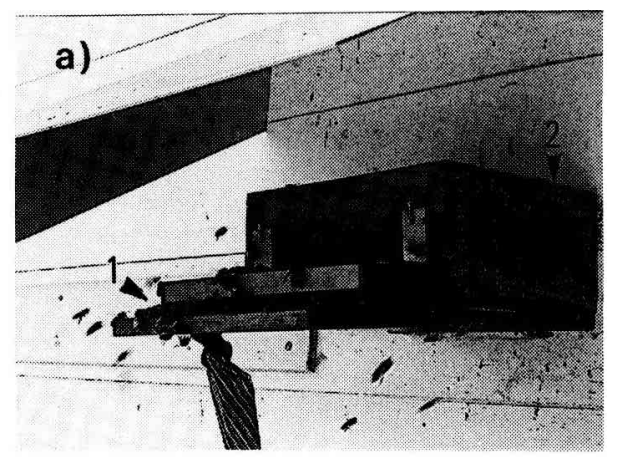

b)

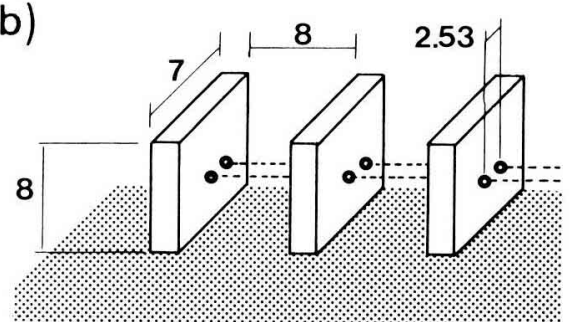

Abb. 1. Bienenzählgerät. 1a) Flugloch mit 16 Kanälen des Zählgeräts (1) und Vorraum aus Drahtgitter (2). 1b) Montage der Lichtschranken (0---) in den Laufkanälen; Masse in Millimeter. 
Ein Decoder tastet den Zustand jeder Lichtschranke ab (Zeit-multiplex-Verfahren). Für die Abtastung aller 16 Lichtschrankenpaare benötigt der Decoder 200 us. Somit werden die Lichtschranken mit $40 \mathrm{~Hz}$ betrieben, so dass eine Bienen mit einer Laufgeschwindigkeit von $1 \mathrm{~m} / \mathrm{sec}$ während ihrer Passage 12,5 mal abgetastet wird. Aus- oder Eingänge werden dann erkannt, wenn die erste und die zweite, rsp. die zweite und die erste Lichtschranke unterbrochen und in der gleichen Reihenfolge wieder freigegeben werden. Um einer möglichen Korrosion entgegenzuwirken, wurden die Schaltungen der Primärelektronik in der Halterung der Lichtschranken in Epoxyharz eingebettet und mit Dellit-Plättchen versiegelt.

\section{Speichern der Daten}

Ein- und Ausgänge werden in der Folge getrennt bearbeitet. Wenn eine vorgewählte Anzahl erfasster Ein- oder Ausgänge erreicht ist, wird eine Marke mit der genauen Uhrzeit (in Hundertstelsekunden) auf einen Zwischenspeicher gespielt. Durch die Möglichkeit, die Anzahl der Ein- oder Ausgänge vorzuwählen, die durch die Zeitmarke repräsentiert sind, kann das Auflösungsvermögen festgelegt werden. Werden Ereignisse einzeln überspielt (vorgewählte Zahl $=1$ ), erhält man die grösstmögliche Auflösung, die Speicherkapazität ist jedoch schnell erschöptt. Damit Tage ohne Flugaktivität z.B. im Winter nicht verloren gehen, wird jeder Tageswechsel mit einem spezielien Code im Zwischenspeicher registriert. Sind 590 Marken im Zwischenspeicher, wird dieser Datenblock auf ein Tonband (Sanyo Microcorder ZE 601) überspielt und der Zwischenspeicher gelöscht. Mit dem blockweisen überspielen wird Strom gespart, so dass das ganze Gerät mit einer 12-V-Autobatterie betrieben werden kann. Zur weiteren Verarbeitung werden die Daten vom Tonband auf Diskette überspielt und in Datenbanken zusammengestellt.

Der Einbau eines Drahtgitterkäfigs zwischen der Wand des Bienenhauses und dem Zähigerät (Abb. 1a) reduzierte die registrierten Bewegungen auf ein Mass, welches mit den Ergebnissen von direkten Beobachtungen übereinstimmte. Wir haben allerdings keine Möglichkeit, anhand der Zählerprotokolle echte Ausflüge von kurzen Aufenthalten auf dem Flugbrett zu unterscheiden. Wo wir von "Flugaktivität” sprechen, meinen wir die gesamten Passagen am Flugloch.

\section{Resultate}

Die gesamten Bienenbewegungen am Flugloch der Jahre 1986 und 1987 des Zählervolkes auf dem Bienenstand der Sektion Bienen, Liebefeld, sind bis auf wenige Tage (defektes Gerät) registriert.

\section{Jahresübersicht 1987}

Im Jahr 1987 wurden insgesamt $10^{\prime} 888^{\prime} 530$ Ausgänge und 10'734'140 Eingänge registriert. Im Januar wurden nur gerade 250 Ausgänge, im Juli dagegen 3'095'200 gezählt. Tabelle I zeigt die Flugaktivität in Abhängigkeit von der Jahreszeit (Abb. 2a) in Abhängigkeit von der Tageszeit am Beispiel des 17. Mai 87.

Setzt man die durchschnittliche tägliche Flugaktivität pro Monat in Beziehung zur Volksstärke, so ergibt sich : Es fliegen umso mehr Bienen aus, je stärker das Volk ist (Abb. 3). 1986 traten die grössten Steigerungen sowohl der Volksstärke als auch der Flugaktivität zwischen Ende Mai und Ende Juni auf. Ab August sank die Flugaktivität, während die Volksstärke in der Erhebung von Mitte August etwas höher lag als in vorangegangenen Messungen. Danach sank die Anzahl Bienen im Volk. 1987 stieg die Volksstärke kontinuierlich an bis zum Maximum Anfang Juni. Die Flugaktivität erreichte ihr Maximum im Juli. (Zwischen Ende Juni und Mitte August 1987 stehen uns keine Daten zur Volksstärke zur Verfügung.) Am 21. Mai 86 wurden pro adulte Biene 3 Larven oder Puppen im Volk gemessen. Unter der Annahme, dass ein Drittel der adulten Bienen Sammelflüge ausführen, ergeben sich für dieses Datum ein Durchschnittswert von 14,2 Flügen pro Flugbiene und Tag. Am 23. Juli 86 (2 Brutzellen pro 3 Bienen, maximale Flugaktivität) liegt 
Tabelle I. Jahresüberblick 1987 : Monatssummen von Ausgängen und Eingängen

\begin{tabular}{lrrrr}
\hline & Ausflüge (A) & Einflüge (E) & Differenz A-E & $A \times 100 / A+E^{\prime}$ \\
\hline Januar & 250 & 200 & 50 & 55,6 \\
Februar & $27^{\prime} 050$ & $26^{\prime} 150$ & 900 & 50,8 \\
März & $80^{\prime} 650$ & $77^{\prime} 700$ & $2^{\prime} 950$ & 50,8 \\
April & $970^{\prime} 450$ & $907^{\prime} 250$ & $63^{\prime} 200$ & 51,7 \\
Mai & $1885^{\prime} 450$ & $1832^{\prime} 600$ & $52^{\prime} 850$ & 50,7 \\
Juni 2 & $2277^{\prime} 200$ & $2154^{\prime} 900$ & $122^{\prime} 300$ & 51,4 \\
Juli 2 & $3095^{\prime} 200$ & $3153^{\prime} 600$ & $-58^{\prime} 400^{2}$ & $49,5^{2}$ \\
August & $1540^{\prime} 000$ & $1569^{\prime} 600$ & $-29^{\prime} 600^{2}$ & $49,5^{2}$ \\
September & $834^{\prime} 800$ & $831^{\prime} 500$ & $3^{\prime} 300$ & 50,1 \\
Oktober & $138^{\prime} 740$ & $137^{\prime} 580$ & $1^{\prime} 160$ & 50,2 \\
November ${ }^{2}$ & $11^{\prime} 080$ & $11^{\prime} 600$ & $-520^{2}$ & $48,9^{2}$ \\
Dezember ${ }^{2}$ & $27^{\prime} 660$ & $31^{\prime} 460$ & $-3^{\prime} 800^{2}$ & $46,8^{2}$ \\
Jan.-Dez. ${ }^{3}$ & $10^{\prime} 888^{\prime} 530$ & $10^{\prime} 734^{\prime} 140$ & $154^{\prime} 390^{3}$ & $50,4^{3}$ \\
\hline & & & & \\
\hline
\end{tabular}

1 Der Wert zeigt an, wieviele Prozent der total registrierten Bewegungen als Ausflüge erfasst wurden $(50 \%=$ gleichviele Ein- wie Ausflüge). ${ }^{2}$ Gerăt teilweise defekt in diesem Monat. 3 Werte von teilweise defektem Gerăt eingeschlossen.

dieser Wert bei 14,6 Flügen pro Biene und Tag. Am 27. April 87 (2 Brutzellen pro Biene) flog eine Flugbiene 8,3 mal pro Tag aus, am 26. Juni 8711,4 mal (2 Brutzellen pro 3 Bienen, maximale Flugaktivität).

\section{Vorspiel}

Nicht alle Ausflüge sind Sammelflüge. Ein Beispiel für das Vorspiel von Bienen sei in Abb. $2 b$ vom 12. September 1987 gezeigt : Bis um $15^{50} \mathrm{~h}$ herrschte geringe
Flugaktivität (10-50 ausfliegende Bienen/Min.). Dann stieg die Aktivität auf maximal 150 Bienen/min. an und fiel 50 min später wieder auf das ursprüngliche Niveau ab. Wetterdaten vom 12. September : Temperatur um $13^{00}: 22^{\circ} \mathrm{C}$, um $19^{00}: 20^{\circ} \mathrm{C}$; leicht bis stark bewölkt; kein Niederschlag; 3,2 h Sonnenscheindauer.

\section{Schwarm}

Am 10.6.87 schwärmte das Zählervolk. Die Aktivitätskurve (Abb. $2 c$ ) zeigt einen 
Bienen/Min.
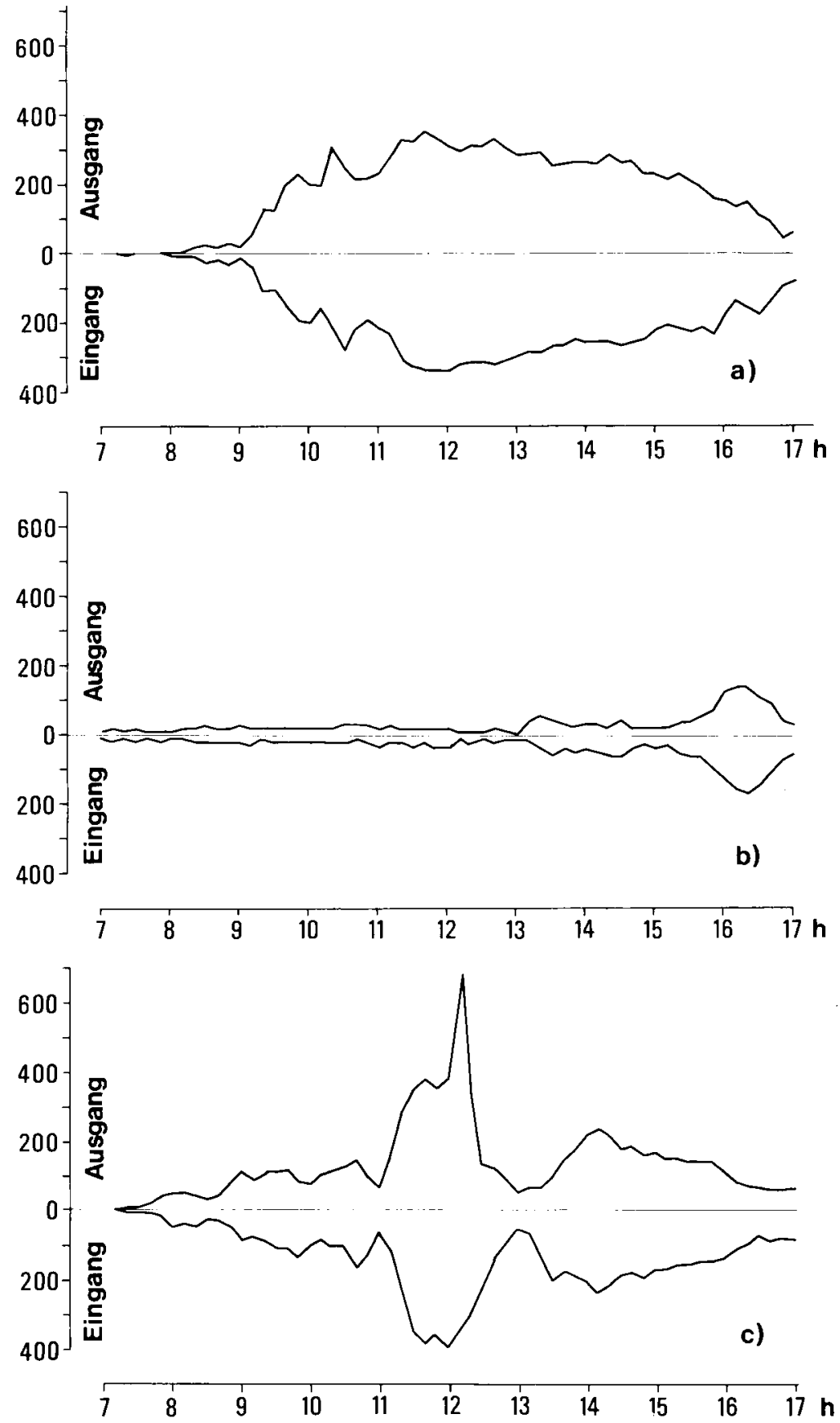

Abb. 2. Flugaktivität im Tagesverlauf. 2a) Flugtag mit hoher Flugaktivität (17. Mai 1987). 2b) Tag mit geringer Flugaktivität, jedoch mit starkem Vorspiel gegen Abend (12. September 1987). 2c) Schwärmendes Volk (10. Juni 1987). 
ungewöhnlichen Verlauf : ab $11 \mathrm{~h}$ stiegen sowohl Aus- wie Eingänge bis um $12 \mathrm{~h}$ stark an. Zwischen $12 \mathrm{~h}$ und $12 \mathrm{~h} 10$ verliessen 3400 Bienen das Volk. Während dieser Zeit wurden bis zu 700 Ausgänge/min. und etwa 400 Eingänge/min. gemessen. Danach sank die Aktivität $a b$, um etwa eine Stunde später wieder auf das gewöhnliche Niveau von ca. 150 Bienen/min. anzusteigen. Als der Schwarm eingefangen wurde, enthielt er an die 10 '000 Bienen $(1,3 \mathrm{~kg})$.

Vergleich der Bienenzähler-Bilanzen mit den natürlichen Abgängen nach Populationsmessung (1986)

Im Jahresdurchschnitt wurden etwa 1\% mehr Aus- als Eingänge registriert. Es traten Tagesschwankungen bis $\mathrm{zu} 10 \%$ auf (55\% Ausgänge, $45 \%$ Eingänge). Da das Gerät in der Regel zuwenig Heimkehrerinnen zählt, fehlen am Abend eines guten Flugtages mit 100'000 Ausflügen laut Messung bis 10'000 Bienen im Stock. Auf Grund der Populationsmessungen wird angenommen, dass der natürliche Abgang des Zählervolkes im Frühsommer durchschnittlich bis 1300 Bienen pro Tag betragen kann. Die Werte nach dem Zählgerät liegen durchwegs höher, als jene der Populationsmessungen (Tabelle II). Insbesondere zwischen Mitte Juni und Mitte August zählt der Bienenzähler mit durchschnittlich 8056 verlorenen Bienen 7,5 mal mehr Verluste, als die Populationsmessungen ergeben (1075 tote Bienen pro Tag).

\section{Diskussion}

\section{Anwendungsmöglichkeiten des Zähl- gerätes}

Fliegen wohl die überlebenden Winterbienen im Frühjahr häufiger aus, weil sie die erste Brutgeneration versorgen müssen, als im Hochsommer, wenn im Verhältnis zur Brutfläche mehr Flugbienen zur Verfügung stehen? Die Berechnungen der Anzahl Ausflüge pro Tag, die eine einzelne Flugbiene ausführt, zeigen, dass dem nicht so ist. 1986 ist zwischen dem Zeitpunkt der grössten Brutmenge pro adulter Biene und jenem der stärksten Flug-

Tabelle II. Vergleich der täglichen Verluste entsprechend nach Populationsmessung (PM) und nach Bienenzählgerät (BZ) (Daten von 1986).

\begin{tabular}{rccc}
\hline Zeitspanne & $\begin{array}{l}\text { Abgang } \\
\text { nach PM } \\
(\text { Bienen/d) }\end{array}$ & $\begin{array}{l}\text { Verlust } \\
\text { nach BZ } \\
\text { (Bienen/d) }\end{array}$ & $\begin{array}{l}\text { Flugaktivität } \\
\text { (Ausflüge/d) }\end{array}$ \\
\hline $9.4 .-30.4$. & 239 & 474 & $10^{\prime} 157$ \\
1.5.-21.5. & 395 & 1257 & $45^{\prime} 252$ \\
$22.5 .-11.6$. & 757 & 876 & $51^{\prime} 350$ \\
12.6.-2.7. & 1257 & 9145 & $128^{\prime} 469$ \\
3.7.-23.7. & 1271 & 6552 & $119^{\prime} 305$ \\
$24.7 .-13.8$. & 696 & 8471 & $89^{\prime} 257$ \\
14.8.-3.9. & 1210 & 2310 & $48^{\prime} 881$ \\
4.9.-24.9. & 347 & 1369 & $27^{\prime} 657$ \\
$9.4 .-24.9$. & 771,5 & 3806,75 & $65^{\prime} 041$ \\
\hline
\end{tabular}


aktivität kein Unterschied festzustellen. 1987 fliegen die Flugbienen pro Tag im Hochsommer öfter aus als zum Zeitpunkt der grössten Brutfläche (Abb. 3). Ob die Dauer oder die eingebrachte Menge pro Sammelflug im Frühjahr und im Sommer unterschiedlich ist, oder ob der Anteil der Sammelflüge an der gesamten Flugaktivität variiert, ist unbekannt. Hier könnten interessante Fragen bearbeitet werden, sofern unsere Ergebnisse, die hier als Beispiel für die Verwendung eines technischen Hilfsmittels stehen, sich durch systematische Untersuchungen erhärten lassen. Wenn zudem die Prozentsätze von Pollen- Nektar- und Wassersammelflügen an der gesamten Flugaktivität als Durchschnittswerte bekannt sind, kann z.B. der "Energiefluss" für ein Volk aufgrund der Flugaktivität für jeden Tag hochgerechnet werden.

Am Schwarmtag (10.6.1987) nahmen sowohl Ein- wie auch Ausgänge bereits eine Stunde vor dem eigentlichen Schwärmen stark zu. Die Dauer, während

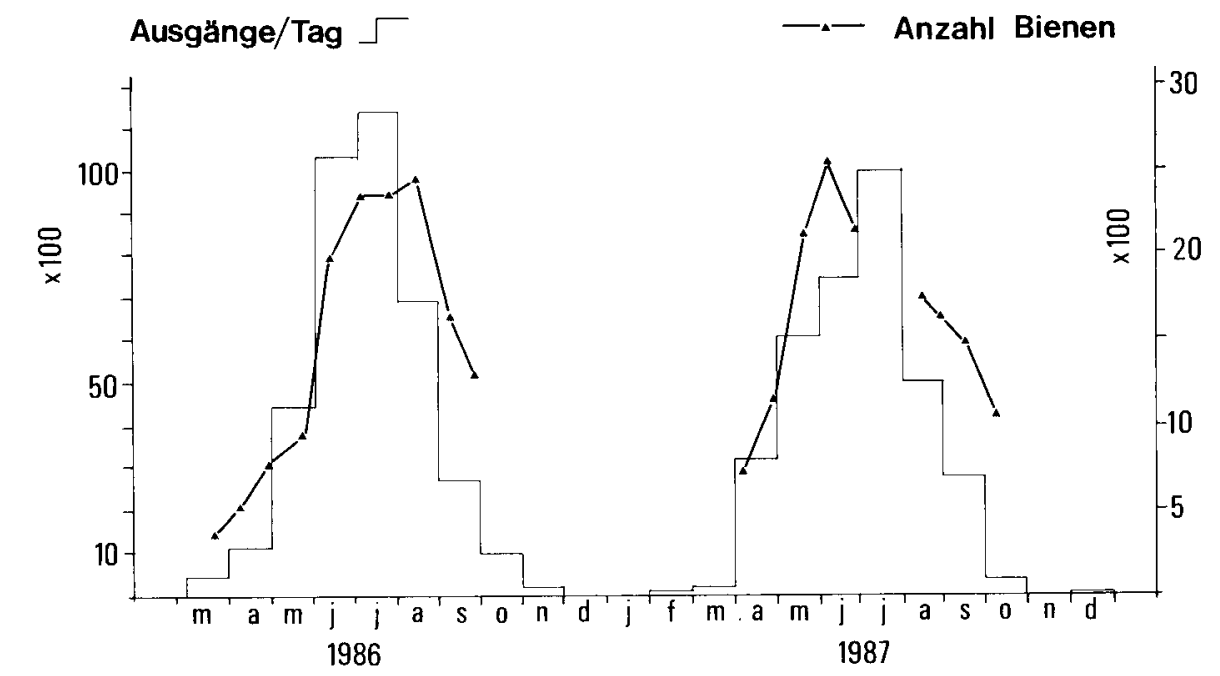

welcher deutlich mehr Bienen das Volk verliessen als zurückkehrten, war mit ca. 20 min relativ kurz gegenüber jenen 2 Stunden, während welchen eine erhöhte allgemeine Aktivität festgestellt wurde. Die Differenz zwischen Bilanz des Zählgerätes (3'400 Bienen) und der Anzahl Bienen nach dem Einfangen des Schwarmes kann wie folgt erklärt werden: Wir wissen aufgrund von Populationsmessungen unmittelbar vor dem Schwärmen und dem Gewicht der eingefangenen Schwärme, dass einem Schwarm sehr oft Bienen aus fremden Völkern zufliegen (Gerig, 1982; Gerig und Imdorf, 1984). Dieses Zufliegen dürfte auch im beschriebenen Fall stattgefunden haben.

\section{Grenzen des Zählgerätes}

Das angestrebte Ziel, den natürlichen Verlust an Flugbienen sowie Verluste bedingt durch Krankheiten oder bienen-

Abb. 3. Vergleich der Flugaktivitäten und Volksstärken der Jahre 1986 und 1987. Die durchschnittliche tägliche Flugaktivität wurde für jeden Monat berechnet (linke Skala). Die dicke Linie zeigt die Volksstärke an (rechte Skala). Zwischen Ende Juni und Mitte August 1987 wurden keine Bestimmungen der Volksstärke durchgeführt. 
Insektizide zu erfassen, wurde wegen ungenügendem Auflösungsvermögen des Zählgerätes bei hoher Flugaktivität nicht erreicht. Die Differenzen "Ausflug minus Einflug" im Zählerprotokoll am Ende eines Tages sind im Durchschnitt 5mal höher, als sie aufgrund der Berechnung nach Populationsmessugen sein sollten. Diese Berechnungen liegen zwischen 240 und 1 300 Bienen pro Tag. Gemessene Sterberaten wurden unseres Wissens bis jetzt nicht publiziert. Da die Bienen in der Mehrzahl ausserhalb des Bienenvolkes sterben, dürfte die Sterberate sehr schwierig zu erfassen sein.

Die Verwendung von Lichtschranken limitiert die Möglichkeiten des Zählgerätes prinzipiell. Die Hauptschwierigkeit ist, dass die Lichtschranke zwei Bienen, die sehr nahe hintereinander laufen, als eine lange Biene erfasst. Eine Vorabklärung hat ergeben, dass bei einem Abstand von $0,5-0,75 \mathrm{~mm}$ von 5 gleichförmig bewegten Objekten in der Regel nur 4 als einzelne Ereignisse erfasst wurden. Wir haben beobachtet, dass die auslaufenden Bienen sich in der Regel schneller bewegen, als die heimkehrenden, die oft in Körperkontakt hintereinander laufen.

Andererseits werden Fehlzählungen dadurch gefördert, dass die ausfliegenden Tiere die vom Flugloch her als heller erscheinenden Flugkanäle des unterteilten Flugloches bevorzugt benützen, während die einfliegenden Tiere die restlichen Kanäle begehen. Heimkehrende Bienen tragen Schmutz auf ihrer Körperoberfläche. Beim Kontakt der Bienen mit Lichtquelle oder -sensor werden diese verschmutzt. Da Bienen sich im Stock reinigen oder von ihren Stockgenossinnen gereinigt werden (z.B. Peng et al., 1987), werden die von den einfliegenden Bienen begangenen Lichtschranken stärker verunreinigt, als die von den ausfliegenden Bienen bevorzugten. Dies zeigt sich darin, dass jene Kanäle am häufigsten gereinigt werden müssen, welche fast ausschliesslich von einfliegenden Bienen benutzt werden.

Eine Möglichkeit unser Zählgerät entscheidend $\mathrm{zu}$ verbessern ist, zusätzlich zur Lichtschrankenmessung eine kapazitative Messung (eine Biene wird als Dielektrikum in einem elektrischen Feld benutzt, z.B. Schwingungskreis) einzubauen. Die Lichtschranken stellen dann die Bewegungsrichtung der Bienen fest, während die Kapazitätsmessung die Anzahl Bienen festhält. Ideal wäre, wenn es gelänge, die Lichtschranken durch zwei kapazitative Messvorrichtungen zu ersetzen. Die Verbesserung scheint unumgänglich, da Bienen oft schubweise das Volk verlassen und wiederum z.T. schubweise zum Flugbrett zurückkehren. Dieses Verhalten tritt nicht nur bei hoher Flugaktivität auf, sondern auch bei schwacher bis mittlerer Aktivität. Deshalb sind Angaben zur Leistungsgrenze, die die Zuverlässigkeit des Gerätes mit Zahlen benennen würden, nicht sinnvoll. Das System "Apicard" (Burill und Dietz, 1973) scheint vom publizierten Zahlenmaterial her mit unserem Gerät vergleichbar. Doch hier, wie auch bei den anderen beschriebenen automatischen Bienenzählgeräten, fehlen Angaben zur Qualität rsp. Aussagekraft der Messwerte.

Trotz der beschriebenen Mängel scheint uns, dass es sich bei diesem Gerät um ein sehr leistungsstarkes handelt. $\mathrm{Da} \beta$ bei solchen Geräten durchschnittliche Fehler im 1\%-Bereich auftreten, ist nicht ungewöhnlich, und eine Erhöhung der Genauigkeit bringt einen hohen Aufwand mit sich. Das Bienenzählgerät kann in der hier beschriebenen Form überall dort eingesetzt werden, wo der Verlauf von Aktivitäts-kurven mehr interessiert als die absoluten Zahlen. 


\begin{abstract}
Summary - An electronic bee counting device : evaluation of its possibilities. An accurate count of exits and entries at the entrance of honeybee hives allows the study of population dynamics and the influences of climate, food availability, diseases and toxic products (particularly insecticides) on bee colonies. A measuring device has been developed to detect and register the exits and entries. Thus, the daily difference between exits and entries can be calculated. This should theoretically correspond to the number of bees that do not return to the hive and presumably die in the field. The aim of this article is to test the reliability of the bee counter and to determine possible applications.
\end{abstract}

To record flight activity, the hive entrance is divided into 16 running channels $(8 \times 8 \times 7 \mathrm{~mm})$, each equipped with a pair of light barriers (Fig. 1). The moving direction (outward or homeward) is determined according to the light beam that is first interrupted by the bee.

In 1987, a bee hive was equipped with the bee counter. During the whole year 10.9 million exits and 10.7 million entries were registered (Table I). For the first time, activity at the hive entrance during swarming could be quantified (Fig. 2c). The errors of the measuring instrument range from $1-2 \%$ (e.g. $51 \%$ exits and $49 \%$ entries). Therefore, with $51^{\prime} 000$ exits a day, there is a deficit or excess of $2^{\prime} 000$ exits. The daily bee loss of the colony calculated from the emergence rate and the registered changes in bee number was $1 ' 300$ bees at maximum and considerably lower than the measured daily differences between exits and entries (Table II). Therefore, the precision of our device is insufficient to register bee losses. The errors might be due to the fact that two bees following each other closely are not always recognized separately by the light barriers. The described bee counter is, however, appropriate for studying daily or seasonal changes in flight activity, swarming (Fig. 2c) and other special phenomena, such as the influence of experimentally restrained access to food sources.

\section{Apis mellifera - actograph - recor- ding device — flight activity - popula- tion dynamics}

\begin{abstract}
Résumé - Un dispositif électronique de comptage des abeilles : évaluation de ses possibilités. Le comptage précis des entrées et des sorties au seuil de la ruche permet d'étudier la dynamique des populations et l'infiuence sur les colonies d'abeilles du climat, des disponibilités en nourriture, des maladies et des produits toxiques (en particulier les insecticides). On a mis au point un dispositif qui détecte et totalise les entrées et les sorties. On peut ainsi calculer la différence journalière entre les sorties et les entrées. Celle-ci correspond théoriquement au nombre d'abeilles qui ne rentrent pas à la ruche et sont probablement mortes dehors. Le but de cet article est de tester la fiabilité du compteur d'abeilles et d'en évaluer les applications possibles.
\end{abstract}

Pour enregistrer l'activité de vol, on a divisé le trou de vol en 16 tubes $(8 \times 8 \times 7$ $\mathrm{mm}$ ), équipés chacun d'une paire de barrières lumineuses (Fig. 1). La direction du mouvement (vers l'extérieur ou vers la ruche) est déterminée par le rayon de lumière coupé en premier par l'abeille.

En 1987, on a équipé une ruche avec ce compteur. Sur l'ensemble de l'année, on a enregistré 10,9 millions de sorties et 10,7 millions d'entrées (Tableau I). Pour la première fois, on a pu quantifier l'activité à l'entrée de la ruche au moment de l'essajmage (Fig. 2c). Les erreurs de mesure de 
l'appareil vont de 1 à $2 \%$ (par exemple $51 \%$ de sorties et $49 \%$ d'entrées). Par conséquent, avec 51000 sorties par jour, il y a un déficit ou un excès de 2000 sorties. La perte journalière d'abeilles d'une colonie calculée d'après le taux d'émergence et les variations enregistrées dans le nombre d'abeilles a été de 1300 abeilles au maximum et considérablement inférieure aux différences journalières mesurées entre les sorties et les entrées (Tableau I). La précision de l'appareil est donc insuffisante pour enregistrer les pertes d'abeilles. Les erreurs peuvent être dues au fait que deux abeilles se suivant de façon très rapprochée ne sont pas toujours reconnues individuellement par les barrières lumineuses. Le compteur d'abeilles décrit convient néanmoins pour étudier les variations saisonnières ou journalières de l'activité de vol, l'essaimage (Fig. 2c) et d'autres phénomènes particuliers tels que l'influence de l'accès restreint aux sources alimentaires lors d'expériences.

\section{Apis mellifera - compteur électro- nique - activité de vol - dynamique de population}

\section{Literaturliste}

Bühlmann G. (1985) Assessing population dynamics in a honey bee colony. Mitt. dtsch. Ges. Allg. Angew. Entomol. 4, 311-316

Bühimann G., Wille M. \& Imdorf A. (1987) Messungen am Flugloch : März-April, Mai-Juni, Juli-August, September-Dezember. Schweiz. Bienenztg. $110(4,6,7,11) 132-140,245-258$, 293-303, 491-500

Burill R.M. \& Dietz A. (1973) An automatic honey bee counting and recording device (Apicard) for possible system analysis of a standard colony. Am. Bee J. 113, 216-218
Chauvin R. (1976) Sur la mesure de l'activité des abeilles au trou de vol d'une ruche à dix cadres. Insectes Soc. 35, 75-82

Danka R.G., Rinderer T.E., Hellmich R.L. \& Collins A.M. (1986) Foraging population sizes of Africanized and European honey bee (Apis mellifera L.) colonies. Apidologie 17 (3), 193202

Danka R.G. \& Gary N.E. (1987) Estimating foraging population of honey bees from individual colonies. J. Econ. Entomol. 80, 544-547

Dietlein D.G. (1985) A method for remote monitoring of activity of honey bee colonies by sound analysis. J. Apic. Res. 24 (3) 176-183

Erickson E.H., Whitefoot L.O. \& Kissinger W.A. (1973) Honey bee : a method of delimiting the complete profile of foraging from colonies. Environ. Entomol. 2 (4), 531-535

Free J.B. \& Preece P.A. (1969) The effect of the size of honey bee colony on its foraging activity. Insectes Soc. 16 (1), 73-78

Gary N.E. (1967) A method for evaluating honey bee flight activity at the hive entrance. J. Econ. Entomol. 60 (1), 102-105

Gerig L. (1982) Welche Bienen schliessen sich dem Schwarm an ? Schweiz. Bienenztg. 105 (3), 122-125

Gerig L. \& Imdorf A. (1984) Entwicklung der Bienenpopulation von zwei Schwarmvölkern und deren Schwärme. Schweiz. Bienenztg. 107 (6), 309-313

Gerig L. \& Bühimann G. (1985) Pseudointoxications of bee colonies - examples of colony breakdown without connection to a pesticide treatment. $3^{\text {rd }}$ Symposium on the harmonisation of methods for testing the toxicity of pesticides to bees, 1985, Rothamsted, GB

Hirschfelder $H$. (1951) Quantitative Untersuchungen zum Polleneintrag der Bienenvöiker. Z. Bienenforsch. 1 (4) 67-77

Imdorf A., Bühlmann G., Gerig L., Kilchenmann V. \& Wille H. (1987) Überprüfung der Schätzmethode zur Ermittlung der Bruttläche und der Anzahl Arbeiterinnen in freifliegenden Bienenvölkern. Apidologie 18 (2), 137-146

Koulichkov N.N. (1971) Method for determining number of foraging bees in the field. Am. Bee J. 111 (7) 268-269

Peng Y.S., Fang Y., Xu S. \& Ge L. (1987) The resistance mechanism of the Asian honey bee Apis cerana to an ectoparasitic mite, Varroa jacobsoni. J. Invertebr. Pathol. 49, 54-60 
Robinson G.E. \& Morse R.A. (1982) Numbers of honey bees that remain out all night. $B e e$ World 63 (3), 117-118

Spangler H.G. (1969) Photoelectrical counting of outgoing and incoming honey bees. $J$. Econ. Entomol. 62 (3), 1183-1184
Spangler H.G. \& Takessian A. (1984) Counting flying honey bees by detecting wing reflectance. Southwest. Entomol. (USA) 2 (1), 35-40

Verbeek B. \& Verbeek R. (1974) Eine Methode zur Registrierung der aus- und einfliegenden Bienen. Apidologie 5 (3), 289-293 